\title{
Anterior segment biometry during accommodation imaged with ultra-long scan depth optical coherence tomography
}

\author{
Chixin Du, MD ${ }^{1,2}$, Meixiao Shen, $\mathrm{MSc}^{2}$, Ming Li, MD³ , Dexi Zhu, $\mathrm{PhD}^{3}$, Michael R. Wang, \\ $\mathrm{PhD}^{4}$, and Jianhua Wang, $\mathbf{M D}, \mathbf{P h D}^{2}$ \\ ${ }^{1}$ Department of Ophthalmology, First Affiliated Hospital, College of Medicine, Zhejiang University, \\ Hangzhou, Zhejiang, China \\ ${ }^{2}$ Bascom Palmer Eye Institute, University of Miami, Miami, FL, USA \\ ${ }^{3}$ School of Ophthalmology and Optometry, Wenzhou Medical College, Wenzhou, Zhejiang, China \\ ${ }^{4}$ Electrical and Computer Engineering, University of Miami, Miami, FL, USA
}

\begin{abstract}
Purpose-To measure by ultra-long scan depth optical coherence tomography (UL-OCT) dimensional changes in the anterior segment of human eyes during accommodation.

Design-Evaluation of diagnostic test or technology.

Participants-Forty-one right eyes of healthy subjects with a mean age of 34 years (range, 2241 years) and a mean refraction of $-2.5 \pm 2.6$ diopters (D) were imaged in two repeated measurements at minimal and maximal accommodation.
\end{abstract}

Methods-A specially adapted designed UL-OCT instrument was used to image from the front surface of the cornea to the back surface of the crystalline lens. Custom software corrected the optical distortion of the images and yielded the biometric measurements. The coefficient of repeatability (COR) and the intraclass correlation coefficient (ICC) were calculated to evaluate the repeatability and reliability.

Main Outcome Measures-Anterior segment parameters and associated repeatability and reliability upon accommodation. The dimensional results included central corneal thickness (CCT), anterior chamber depth and width (ACD, ACW), pupil diameter (PD), lens thickness (LT), anterior segment length $(\mathrm{ASL}=\mathrm{ACD}+\mathrm{LT})$, lens central position $(\mathrm{LCP}=\mathrm{ACD}+1 / 2 \mathrm{LT})$ and horizontal radii of the lens anterior and posterior surface curvatures (LAC, LPC).

() 2012 American Academy of Ophthalmology, Inc. Published by Elsevier Inc. All rights reserved.

Corresponding Author: Jianhua Wang, MD, PhD, Mailing address: Bascom Palmer Eye Institute, University of Miami, Miller School of Medicine, 1638 NW 10th Avenue, McKnight Building - Room 202A, Miami, FL, USA 33136, Tel: (305) 482-5010, jwang3@med.miami.edu.

Address for reprints: Bascom Palmer Eye Institute, University of Miami, Miller School of Medicine, 1638 NW 10th Avenue, McKnight Building - Room 202A, Miami, FL, USA 33136

Publisher's Disclaimer: This is a PDF file of an unedited manuscript that has been accepted for publication. As a service to our customers we are providing this early version of the manuscript. The manuscript will undergo copyediting, typesetting, and review of the resulting proof before it is published in its final citable form. Please note that during the production process errors may be discovered which could affect the content, and all legal disclaimers that apply to the journal pertain.

Meeting Presentation: A part of the method section about special designed ultra-long OCT setting has been reported as paper representation at ARVO's 2011 annual meeting.

Financial Disclosures: All authors have no proprietary interest in any materials or methods described within this article. No conflicting relationship exists for any authors. 
Results-Repeated measurements of each variable within each accommodative state did not differ significantly (P>0.05). The CORs and ICCs for CCT, ACW, ACD, LT, LCP, and ASL were excellent ( $1.2 \%$ to $3.59 \%$ and 0.998 to 0.877 , respectively). They were higher for PD ( $18.90 \%$ to $21.63 \%$ and 0.880 to 0.874 , respectively), and moderate for LAC and LPC (34.86\% to $42.72 \%$ and 0.669 to 0.251 , respectively) in the two accommodative states. Compared to minimal accommodation, PD, ACD, LAC, LPC, and LCP decreased and LT and ASL increased significantly at maximal accommodation $(\mathrm{P}<0.05)$, while $\mathrm{CCT}$ and $\mathrm{ACW}$ did not change $(\mathrm{P}>0.05)$.

Conclusions-UL-OCT measured changes in anterior segment dimensions during accommodation with good repeatability and reliability. During accommodation, the back surface of the lens became steeper as the lens moved forward.

\section{Keywords}

Anterior segment biometry; accommodation; optical coherence tomography; quantitative analysis

Understanding the mechanism of accommodation has attracted great interest among both ophthalmic and optometric researchers. ${ }^{1-3}$ Special attention has been given to work that might enable the recovery of accommodative power for millions of elderly people and for those who have undergone cataract surgery with accommodative intraocular lens (IOL) implantation. Arguments regarding the accommodative mechanisms are still vehement since the debut of Helmholtz's theory of accommodation which has been generally accepted by most ophthalmologists. ${ }^{4}$ A likely explanation for these disagreements is measurement error due to the low resolution imaging modalities or the subjective methods previously used. 5,6 Recently, several techniques have been developed for imaging the anterior segment of the eye in vivo. These modalities, including Scheimpflug photography, ${ }^{2} \mathrm{~A} / \mathrm{B}$ scan ultrasound, ${ }^{7}$ ultrasound biomicroscopy, ${ }^{8}$ slit scanning topography, and Purkinje imaging, ${ }^{9}$ have enabled the objective assessment of structural changes that occur during accommodation. ${ }^{10}$ With these technologies, the dynamic accommodative process can only be observed indirectly by stimulating the contralateral eye. Furthermore, these techniques are susceptible to errors associated with anisometropia or ocular asymmetry.

Optical coherence tomography (OCT) has evolved rapidly for non-invasive cross-sectional imaging of the eye, and it can now produce static and dynamic anterior segment images. Currently it has widespread use in clinical diagnosis and in the treatment of anterior segment diseases. ${ }^{11-14}$ It is also capable of exploring changes in anterior chamber dimensions and lens thickness with aging and accommodation. ${ }^{5,15-17}$ However, simultaneous imaging of the whole anterior segment, including the cornea, iris, anterior chamber, and crystalline lens, cannot be accomplished by the commercially available OCT instruments because the imaging depth, $\sim 6 \mathrm{~mm}$, is too short, and the imaging speed, $\sim 4,000 \mathrm{~A}$-scans $/ \mathrm{sec}$, is too slow. For instruments with imaging speeds of 20,000-40,000 A-scans/sec, the imaging depth is less than $8 \mathrm{~mm}$.

Based on our prior prototypes of spectral domain (SD)-OCT instruments, ${ }^{18,19}$ we have now developed a custom ultra-long scan depth OCT (UL-OCT) system that achieves extended scan depths of more than $11 \mathrm{~mm}$. With this capacity, simultaneous imaging of the entire human anterior segment from the front of the cornea through the crystalline lens is now possible. In this report, we evaluated the repeatability and reliability of the new system. We have also documented accommodative changes of anterior segment dimensions.

\section{Methods}

This study was approved for human research by the Institutional Review Board of the University of Miami. Informed consent was obtained from each subject, and the study 
complied with Health Insurance Portability and Accountability Act. Each subject was treated in accordance with the tenets of the Declaration of Helsinki. All subjects were recruited from the University of Miami from October 2011 to June 2012. Exclusion criteria were best corrected distance visual acuity worse than 20/25; nystagmus; anterior or posterior segment pathology; medication that might affect pupil constriction or accommodation; history of laser treatment, trauma, or eye surgery; or systemic disease. The right eyes of 41 participants (17 males and 24 females) with a mean \pm standard deviation age of $33.5 \pm 6.9$ years and a mean refraction of $-2.5 \pm 2.6$ diopters (D) were enrolled in this study.

Utilizing a fiber-based Michelson interferometer, the UL-OCT system was constructed with a superluminescent diode laser-based light source (InPhenix, IPSDD0808, Livermore, CA, USA) with center wavelength of $840 \mathrm{~nm}$ and full-width at half maximum bandwidth of 50 $\mathrm{nm}$. The light was focused on the anterior segment, and the total exposure power at the surface of the cornea was below $1.40 \mathrm{~mW}$. Because the light was focused on the anterior segment and not the retina, the exposure power was much less than the safe cut-off value for long exposure to the eye according to ANSI Z136.1. ${ }^{20}$ The scan speed was set to $4,000 \mathrm{~A}$ scans/sec, a limitation imposed by the charged-couple device (CCD) line scan camera (2,048 pixels; Aviiva-SM2010; E2V Technologies, Tarrytown, NY, USA).

The scanning and optical delivery system was built on a modified slit-lamp with a video camera for monitoring and imaging the eye. The integration time was set to $216 \mu \mathrm{s}$. An internal fixation target "E" displayed on a miniature liquid crystal display with black background was set about $20 \mathrm{~cm}$ away from the test eye. The lens, about $10 \mathrm{~cm}$ in front of the fixation target, was adjustable; therefore the amplitude of the accommodation was controlled subjectively based on the assumption that the subject could see the target as clearly as possible. The scan depth of the system was $11.865 \mathrm{~mm}$. An alternative placement of the zero-delay line on the top and bottom in the reference arm was specially designed to acquire multiple images in one scan. Image enhancement was realized by overlapping the two acquired images. This method was used to compensate the drop of signal-to-noise ratio through the entire image depth. The resolution of the UL-OCT system was measured as 10.4 $\mu \mathrm{m}$ axially and approximately $15 \mu \mathrm{m}$ transversely for 2,048 pixels in the eye. The scan width was set at $12 \mathrm{~mm}$.

All OCT images were obtained in a normal examination room with monitored environmental settings of temperature $\left(15-25^{\circ} \mathrm{C}\right)$ and humidity $(30-50 \%)$, and the light was dimmed to avoid possible pupillary constriction. Each subject sat in front of the slit-lamp and looked forward at the internal fixation target "E". Positive or negative lenses were placed in the lens holder to compensate for the subject's spherical ametropia for near equivalent spherical refractive correction. After adjusting the fixation to ensure the perfect alignment for image acquisition, a cross-sectional scan at the horizontal meridian across the corneal apex was obtained at the minimal accommodation state. Afterwards, negative lenses were gradually added to the lens holder to stimulate the maximal physiologic accommodation until the subject reported that the target became blurred. Two consecutive images of each eye were acquired at each different accommodative state, and the mean of the two results was taken for further statistical analysis.

The two-dimensional cross-sectional images (B-scans) consisted of 2,048 line scans (Ascans), with 2,048 points per A-scan. The raw images were exported and then overlaid in registration with the lens anterior surface. The boundaries of the cornea and lens were outlined semi-manually in the construction image using custom software. ${ }^{21}$ Nine anterior segment parameters were determined with the post-process constructed images (Fig. 1). On the cross-sectional image with the corneal vertex reflection, the anterior chamber width (ACW) was measured as the distance from angle to angle (ATA). On the same cross- 
sectional image, to reduce the measurement error resulting from the tilt, a line was drawn along the ATA distance. From that line a perpendicular projection extending forward from the median point through the cornea was drawn. Central corneal thickness (CCT), anterior chamber depth (ACD), lens thickness (LT) ${ }^{11}$ anterior segment length $(\mathrm{ASL}=\mathrm{ACD}+\mathrm{LT}),{ }^{22}$ and lens central position $(\mathrm{LCP}=\mathrm{ACD}+1 / 2 \mathrm{LT})$ were measured along this perpendicular line. Additionally, the pupil diameter (PD), horizontal radii of the lens anterior and posterior surface curvatures (LAC, LPC) were all described and calculated on the same cross-section. A custom algorithm based on Snell's principle ${ }^{23}$ was used for correction of optical distortion of each boundary. This algorithm incorporated wrapping of the refractive indices for the cornea (1.378), aqueous humor (1.33), and the crystalline lens (1.416). ${ }^{23,24}$

An ocular imaging eye model (OEMI-7, Ocular ${ }^{\circledR}$ Instruments Inc., Bellevue, WA, USA) with a polymethyl methacrylate (PMMA) cornea and IOL was used to validate the optical correction of the algorithm (Fig. 2). Using the refractive index of PMMA (1.485 at $840 \mathrm{~nm}$ light wavelength), the anterior and posterior curvature measurements of the model cornea and the IOL were determined. These measurements were compared with similar ones of the model eye made by a Zygo Interferometric Optical Surface Profiler (Zygo ${ }^{\mathrm{TM}}$ Corporation, Middlefield, CT, USA).

Statistical Procedures for the Social Sciences (SPSS for Windows 16.0, SPSS Inc., Chicago, IL, USA) was used for descriptive statistics and data analysis. All data were presented as means \pm standard deviations. After determining that the data for each variable was normally distributed, paired t-tests were used to compare the repeated measures of the same accommodative state and the two different accommodative states. P-values less than 0.05 were considered statistically significant. The coefficient of repeatability (COR) was calculated as two standard deviations of the differences between measurements at the same accommodative state divided by the average of each pair of measurements and expressed as a percentage. The intraclass correlation coefficient (ICC) for absolute agreement was calculated to assess the reliability of the repeated measurements.

\section{Results}

The UL-OCT measured radii for a model eye with a PMMA cornea and IOL matched well with those determined by surface optical interferometry (Table 1). For human eyes, the simultaneously acquired raw images showed the whole anterior segment of the eye from the anterior corneal surface through the lens (Fig. 1). The anterior and posterior surfaces of the crystalline lens and the lens structural morphology were clearly visualized.

\section{Repeatability and reliability of anterior segment biometry}

For all anterior segment dimensions, there were no significant differences between the two repeated measurements of the same accommodative state (paired t-tests, all p-values $<0.05$, Table 2, available at http://aaojournal.org). The average of the two measurements for each accommodative state was used to determine if there were differences between two states. The CORs for CCT, ACW, ACD, LT, LCP, and ASL were excellent, ranging from $1.23 \%$ to $3.59 \%$ in the minimal and maximal accommodative states. The CORs for PD were relatively high, $18.90 \%$ and $21.63 \%$ in minimal and maximal accommodative states respectively. The CORs for LAC and LPC ranged from $34.86 \%$ to $42.70 \%$ in both accommodative states (Table 2, available at http://aaojournal.org). The ICCs for CCT, ACD, LT, LCP, and ASL were very high, varying between 0.969 and 0.998 . They were slightly lower for PD and ACW, 0.874 to 0.925 , and showed moderate and fair reliability for LAC and LPC, 0.251 to 0.669 (Table 2, available at http://aaojournal.org). 


\section{Accommodative changes of anterior segment biometry}

The CCT and ACW did not change between accommodative states (paired t-test, $\mathrm{p}=0.0602$, 0.8345 , respectively, Table 3, available at http://aaojournal.org). In contrast, decreases in PD, LAC, and LPC ( $\mathrm{p}<0.05$ each) were accompanied by increases of LT $(\mathrm{p}<0.05)$. Consequently the ASL increased, and LCP moved forward (anteriorly) during accommodation (paired t-tests, all $\mathrm{p}<0.05$, Table 3, Fig. 3).

\section{Discussion}

Imaging the entire anterior segment simultaneously from the cornea through the crystalline lens, which is optically accessible only through the pupil, is a challenge for OCT. Commercially available anterior segment OCT instruments and some prototypes have been used to study accommodation. ${ }^{15,17}$ However, with these instruments, only a portion of the anterior segment could be imaged, and the posterior surface of the lens was often not imaged. With the development of complicated phase shifts, long scan depth imaging was achieved, which then made it possible to image the entire anterior segment. ${ }^{25,26}$ Grulkowski et al. used a high speed complementary metal-oxide semiconductor (CMOS) camera and eliminated complex ambiguity to image the anterior segment of the human eye. ${ }^{17}$ This method has not been widely used yet, possibly due to its complexity and the high cost of the setup. OCT imagery based on a swept light source also achieves long scan depths. ${ }^{27,28} \mathrm{We}$ previously developed a dual-channel dual-focus OCT system for imaging structural changes that occur during accommodation. ${ }^{19}$ However that system required two spectrometers to achieve long scan depths, and the attenuation of 50\% of the reflected light resulted in low image contrast. In the present study, we used an alternative approach that utilized a modified SD-OCT instrument with a single spectrometer. Image enhancement was realized by employing a novel image acquisition and processing technique. This method overlaid two images with the alternative placement of the zero-delay line on the top and bottom in the reference arm. This technique not only sustained the high contrast of the image but also compensated the drop of signal-to-noise ratio. Consequently, the scan depth was extended without removing complex conjugated image ambiguity (Du C, et al. IOVS 2011, 52, ARVO E-Abstract 3023). Thus, this method is able to image the entire anterior segment along the optical axis through the pupil. The system may be readily accessible for use in clinics due to its cost-effective setup and contrast enhancement.

Testing the repeatability of measurements is critical for interpretation of structural changes that occur during accommodation. There is no information available about the repeatability of other methods for acquiring these measurements. Because accommodation is a dynamic process, knowing the repeatability at each accommodative state is important. In the present study, we showed good repeatability between the two measurements in each accommodative state for all of the anterior segment dimensions except LAC and LPC. The difference of $0.98 \%$ in CCT and $1.50 \%$ in ACW between the minimal and maximal accommodative states also demonstrated that the system was repeatable and reliable enough to investigate precise and sensitive lens changes during accommodation. However, the scan area of the lens was limited by the size of the pupil, especially the smaller aperture during accommodation. This could have affected the lens curvature measurements. Because the lens surface may be aspheric, ${ }^{29}$ an error may have been introduced during the curve fitting procedure. The dynamic changes that occur during accommodation may also have induced errors in the lens curvature measurements. So for both lens curvatures, the CORs and ICCs were low. The ACD, ACW, LT, and LAC values were in good agreement with previous studies. ${ }^{15,29-32}$ The LPC was about $5 \mathrm{~mm}$, which is slightly smaller than the value recently report by magnetic resonance imaging (MRI). ${ }^{29}$ In addition to image calibration, measurement errors may be due to the small scan area of the lens (about $5 \mathrm{~mm}$ ) in the present study, compared to $10 \mathrm{~mm}$ of the scan region with MRI. 
Our findings on the dimensional changes that occurred during accommodation support the classic theory by Helmholtz, ${ }^{1}$ Fincham, ${ }^{4}$ and others. ${ }^{13,30,31}$ During accommodation, the anterior surface of the lens became steeper and moved forward, causing the anterior chamber to become more shallow. Interestingly, the back surface of the lens also became steeper. The steepening of anterior lens curvature was greater than that of the posterior curvature, suggesting that the anterior surface has a greater role during accommodation. However, though the magnitude of the posterior surface changes was less than the anterior surface, the changes in posterior curvature may be important, especially in elderly people whose lenses have a relative higher refractive index.

There are few reports regarding changes in the posterior lens surface during accommodation due to limitation of in vivo imaging technology. Among the studies that do address this issue, the results are contradictory. ${ }^{5,6,33}$ For instance, the lens was reported to be pushed towards the cornea during accommodation because of the hydraulic pressure of the vitreous. ${ }^{33}$ However other studies suggested that the posterior lens surface moved backward during accommodation. ${ }^{5,6} \mathrm{~A}$ recent report using 3D MRI showed that the lens thickened and both surface radii decreased during accommodation. ${ }^{32}$ Dubbelman et al., ${ }^{30}$ using Scheimpflug imaging, reported a decrease in both anterior and posterior lens radii. The authors found the increase in lens thickness with accommodation was higher than the decrease in the anterior chamber depth, indicating that the posterior lens surface moves backwards with accommodation. By partial coherence interferometry, Tsorbatzoglou et al. ${ }^{22}$ found that the ASL increased, indicating that the lens posterior surface moved backward. They postulated that the anterior shifting of the lens may also participate in the accommodative response. Our data were similar to those of Tsorbatzoglou et al. ${ }^{22}$ with respect to the changes of lens shape and position during accommodation. Measuring the LCP provides an easy way for tracking the movement of the lens because it clarifies the change of lens position during accommodation while the anterior surface moves forward and the posterior surface moves backward. The LCP may useful in the detection of IOL biodynamic changes in the posterior chamber and beneficial to accommodative IOL design.

There are some limitations of this methodology. First, using the iris image and the apex for positioning the eye may induce an alignment error. Real-time views of horizontal and vertical scans for alignment may help in minimizing the error. Second, the scanning speed was set at 4,000 A-scans/sec based on the CCD line camera imaging speed limit. The use of a CMOS camera in a SD-OCT system or swept light source may make the system faster for three dimensional scanning.

In conclusion, the UL-OCT system successfully measured the dimensional changes of the anterior segment with good repeatability and reliability during accommodation. During accommodation, the back surface of the lens became steeper as the lens moved forward.

\section{Supplementary Material}

Refer to Web version on PubMed Central for supplementary material.

\section{Acknowledgments}

We thank Britt Bromberg, PhD, Xenofile Editing, for providing critical editing services for this manuscript.

Grant/financial support: This study was supported by research grants from NIH Center Grant P30 EY014801, Research to Prevent Blindness (RPB). The sponsor or funding organization had no role in the design or conduct of this research. 


\section{References}

1. Glasser A, Kaufman PL. The mechanism of accommodation in primates. Ophthalmology. 1999; 106:863-872. [PubMed: 10328382]

2. Koretz JF, Cook CA, Kaufman PL. Accommodation and presbyopia in the human eye. Changes in the anterior segment and crystalline lens with focus. Invest Ophthalmol Vis Sci. 1997; 38:569-578. [PubMed: 9071209]

3. Charman WN. The eye in focus: accommodation and presbyopia. Clin Exp Optom. 2008; 91:207225. [PubMed: 18336584]

4. Fincham EF. The proportion of ciliary muscular force required for accommodation. J Physiol. 1955; 128:99-112. [PubMed: 14368577]

5. Baikoff G, Lutun E, Wei J, Ferraz C. Anterior chamber optical coherence tomography study of human natural accommodation in a 19-year-old albino. J Cataract Refract Surg. 2004; 30:696-701. [PubMed: 15050270]

6. Beauchamp R, Mitchell B. Ultrasound measures of vitreous chamber depth during ocular accommodation. Am J Optom Physiol Opt. 1985; 62:523-532. [PubMed: 4037058]

7. Kirschkamp T, Dunne M, Barry JC. Phakometric measurement of ocular surface radii of curvature, axial separations and alignment in relaxed and accommodated human eyes. Ophthalmic Physiol Opt. 2004; 24:65-73. [PubMed: 15005670]

8. Ishikawa H, Schuman JS. Anterior segment imaging: ultrasound biomicroscopy. Ophthalmol Clin North Am. 2004; 17:7-20. [PubMed: 15102510]

9. Rosales P, Dubbelman M, Marcos S, van der HR. Crystalline lens radii of curvature from Purkinje and Scheimpflug imaging. J Vis. 2006; 6:1057-1067. [PubMed: 17132077]

10. Wolffsohn JS, Davies LN. Advances in anterior segment imaging. Curr Opin Ophthalmol. 2007; 18:32-38. [PubMed: 17159445]

11. Radhakrishnan S, Goldsmith J, Huang D, et al. Comparison of optical coherence tomography and ultrasound biomicroscopy for detection of narrow anterior chamber angles. Arch Ophthalmol. 2005; 123:1053-1059. [PubMed: 16087837]

12. Goldsmith JA, Li Y, Chalita MR, et al. Anterior chamber width measurement by high-speed optical coherence tomography. Ophthalmology. 2005; 112:238-244. [PubMed: 15691557]

13. Baikoff G, Jitsuo JH, Bourgeon G. Measurement of the internal diameter and depth of the anterior chamber: IOL Master versus anterior chamber optical coherence tomographer. J Cataract Refract Surg. 2005; 31:1722-1728. [PubMed: 16246775]

14. Hurmeric V, Yoo SH, Fishler J, et al. In vivo structural characteristics of the femtosecond LASIKinduced opaque bubble layers with ultrahigh-resolution SD-OCT. Ophthalmic Surg Lasers Imaging. 2010; 41(Suppl):S109-S113. [PubMed: 21117595]

15. Richdale K, Bullimore MA, Zadnik K. Lens thickness with age and accommodation by optical coherence tomography. Ophthalmic Physiol Opt. 2008; 28:441-447. [PubMed: 18761481]

16. Yan PS, Lin HT, Wang QL, Zhang ZP. Anterior segment variations with age and accommodation demonstrated by slit-lamp-adapted optical coherence tomography. Ophthalmology. 2010; 117:2301-2307. [PubMed: 20591484]

17. Grulkowski I, Gora M, Szkulmowski M, et al. Anterior segment imaging with Spectral OCT system using a high-speed CMOS camera. Opt Express. 2009; 17:4842-4858. [PubMed: 19293916]

18. Shen M, Wang MR, Yuan Y, et al. SD-OCT with prolonged scan depth for imaging the anterior segment of the eye. Ophthalmic Surg Lasers Imaging. 2010; 41(Suppl):S65-S69. [PubMed: 21117604]

19. Zhou C, Wang J, Jiao S. Dual channel dual focus optical coherence tomography for imaging accommodation of the eye. Opt Express. 2009; 17:8947-8955. [PubMed: 19466144]

20. American National Standards Institute. ANSI Z136.1-2007, American National Standard for safe use of lasers. Washington: American National Standards Institute Inc; 2007.

21. Tao A, Wang J, Chen Q, et al. Topographic thickness of Bowman's layer determined by ultra-high resolution spectral domain-optical coherence tomography. Invest Ophthalmol Vis Sci. 2011; 52:3901-3907. [PubMed: 21460260] 
22. Tsorbatzoglou A, Nemeth G, Szell N, et al. Anterior segment changes with age and during accommodation measured with partial coherence interferometry. J Cataract Refract Surg. 2007; 33:1597-1601. [PubMed: 17720076]

23. Zhao M, Kuo AN, Izatt JA. 3D refraction correction and extraction of clinical parameters from spectral domain optical coherence tomography of the cornea. Opt Express. 2010; 18:8923-8936. [PubMed: 20588737]

24. Uhlhorn SR, Borja D, Manns F, Parel JM. Refractive index measurement of the isolated crystalline lens using optical coherence tomography. Vision Res. 2008; 48:2732-2738. [PubMed: 18824191]

25. Jungwirth J, Baumann B, Pircher M, et al. Extended in vivo anterior eye-segment imaging with full-range complex spectral domain optical coherence tomography. J Biomed Opt. 2009; 14:050501. [PubMed: 19895097]

26. Kerbage C, Lim H, Sun W, et al. Large depth-high resolution full 3D imaging of the anterior segments of the eye using high speed optical frequency domain imaging. Opt Express. 2007; 15:7117-7125. [PubMed: 19547029]

27. Potsaid B, Baumann B, Huang D, et al. Ultrahigh speed 1050nm swept source/Fourier domain OCT retinal and anterior segment imaging at 100,000 to 400,000 axial scans per second. Opt Express. 2010; 18:20029-20048. [PubMed: 20940894]

28. Furukawa H, Hiro-Oka H, Satoh N, et al. Full-range imaging of eye accommodation by high-speed long-depth range optical frequency domain imaging. Biomed Opt Express. 2010; 1:1491-1501. [PubMed: 21258564]

29. Sanjeev, Kasthurirangan; Markwell, EL., et al. MRI study of the changes in crystalline lens shape with accommodation and aging in humans. J vis. 2011; 11:1-16.

30. Dubbelman M, Van der Heijde GL, Weeber HA. Change in shape of the aging human crystalline lens with accommodation. Vision Res. 2005; 45:117-132. [PubMed: 15571742]

31. Rosales P, Wendt M, Marcos S, Glasser A. Changes in crystalline lens radii of curvature and lens tilt and decentration during dynamic accommodation in rhesus monkeys. J Vis. 2008; 8:18.118.12. [PubMed: 18318621]

32. Sheppard AL, Evans CJ, Singh KD, et al. Three-dimensional magnetic resonance imaging of the phakic crystalline lens during accommodation. Invest Ophthalmol Vis Sci. 2011; 52:3689-3697. [PubMed: 21296812]

33. Coleman DJ. Unified model for accommodative mechanism. Am J Ophthalmol. 1970; 69:10631079. [PubMed: 5423772] 


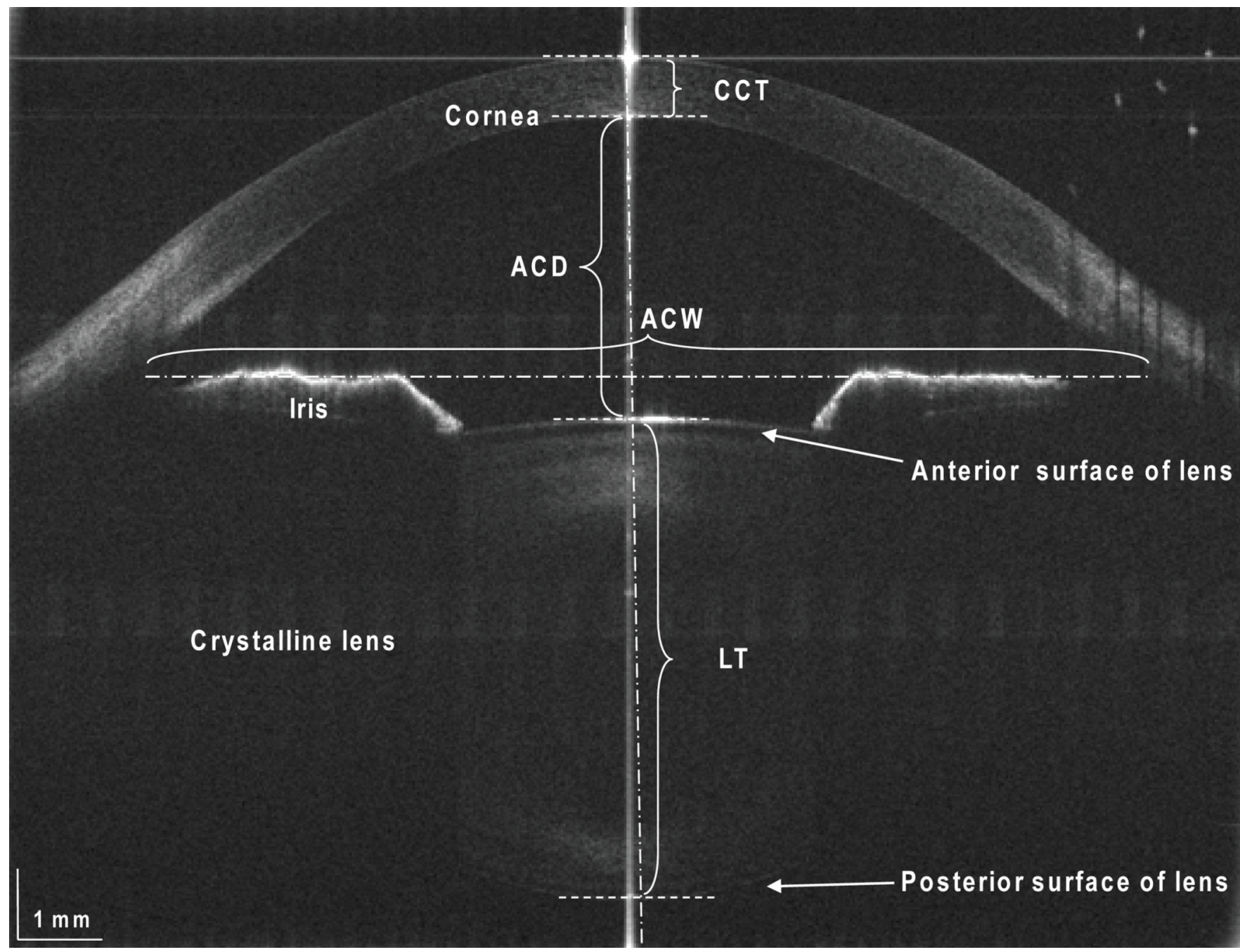

Figure 1.

Raw constructed images of the anterior segment. The constructed image clearly showed the whole anterior segment of the eye, including cornea, anterior chamber, iris, anterior, and the full thickness of the crystalline lens. Pixels: $2048 \times 2048$; image range: $11.4 \mathrm{~mm}(\mathrm{depth}) \times 12$ $\mathrm{mm}$ (width). On the two-dimensional cross-sectional images with the corneal vertex reflection, the anterior chamber width $(\mathrm{ACW})$ was measured as the distance from angle to angle. A perpendicular line was drawn from angle to angle and extended forward from the median point through the cornea. Central corneal thickness (CCT), anterior chamber depth (ACD), and lens thickness (LT) were measured along this perpendicular line. 


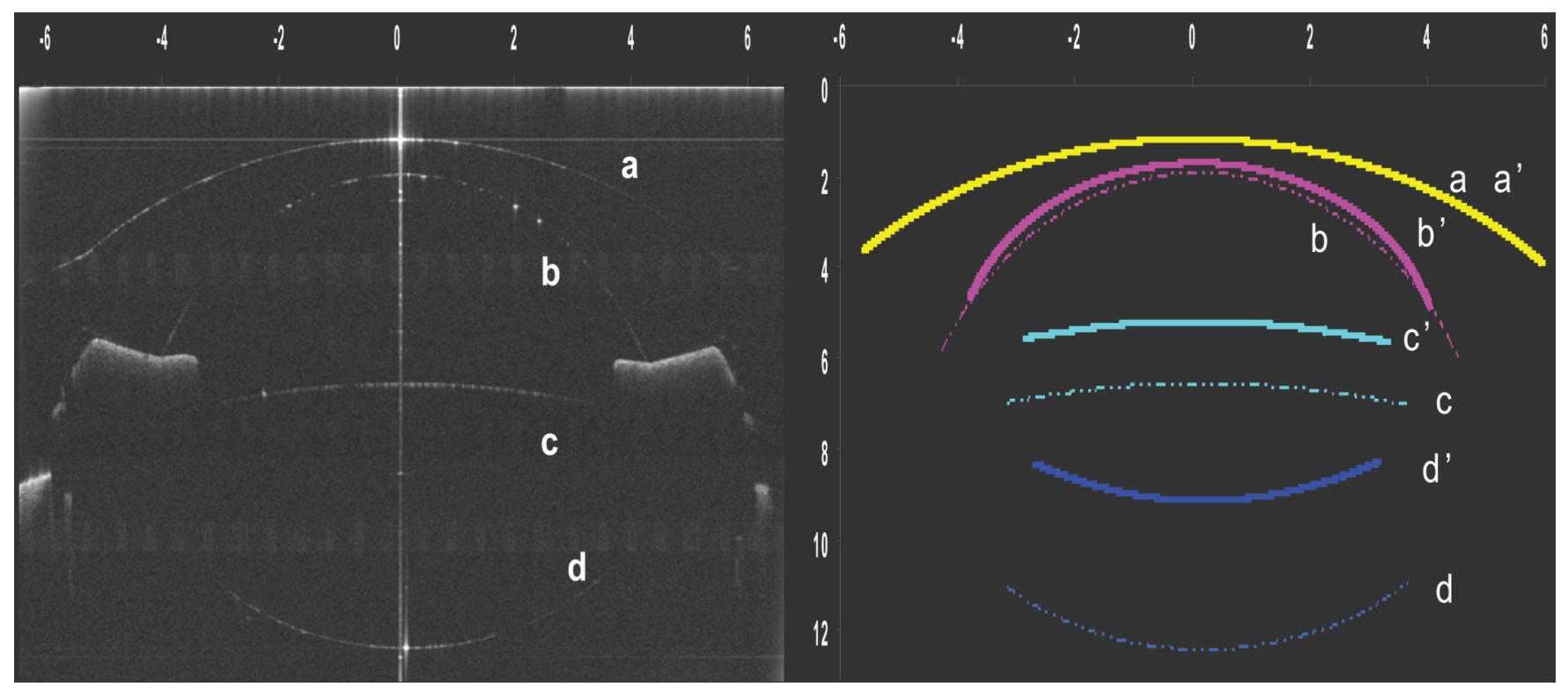

Figure 2.

(Left) Raw constructed image of the model eye. a, anterior corneal surface; b, posterior corneal surface; c, anterior lens surface; d, posterior lens surface. (Right) Profile of all surfaces of the model eye. Solid lines represent raw surfaces that were measured: a, anterior corneal surface; b, posterior corneal surface; c, anterior lens surface; d, posterior lens surface. Dashed lines b', c' and d' represent corrected surfaces that were measured and corresponded to $\mathrm{b}, \mathrm{c}$ and d respectively. 

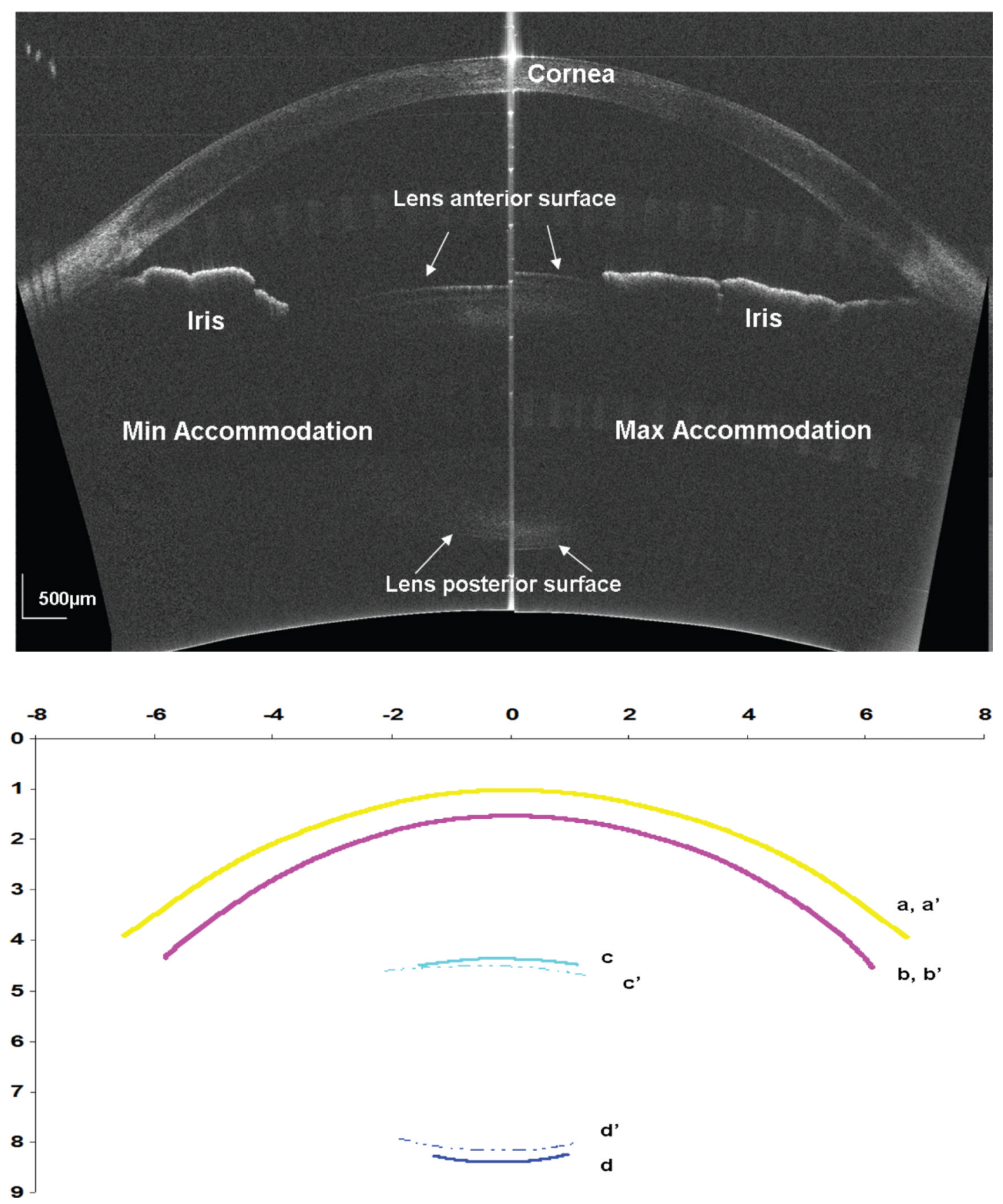

Figure 3.

(Upper) Corrected images at the minimal (left) and maximal (right) accommodative states in a 28-year-old male with -6.00 diopter (D) myopia. This image was corrected only at both corneal surfaces. (Lower) Profile of all anterior segment surface corrected measurements of the same eye at minimal accommodation (dashed lines a', b', c', and d') and maximal accommodation (solid lines a, b, c and d). 
Table 1

Interferometry and UL-OCT measured radii for a model eye with a PMMA cornea and IOL.

\begin{tabular}{ccc}
\hline Radii $^{*}$ & Zygo $^{\text {TM }}$ interferometry & OCT with correction ${ }^{* *}$ \\
\hline PMMA Cornea & & \\
Anterior & 7.68 & 7.67 \\
Posterior & 4.08 & 4.04 \\
\hline Intraocular lens & & \\
Anterior & 12.00 & 12.11 \\
Posterior & 5.91 & 5.85 \\
\hline
\end{tabular}

* Radii were measured in millimeters.

All UL-OCT measured radii were corrected in the optical distortion correction algorithm using the refractive index of PMMA, 1.485 at the wavelength $840 \mathrm{~nm}$.

UL-OCT, ultra-long scan depth optical coherence tomography; IOL, Intraocular lens; PMMA, polymethyl methacrylate. 